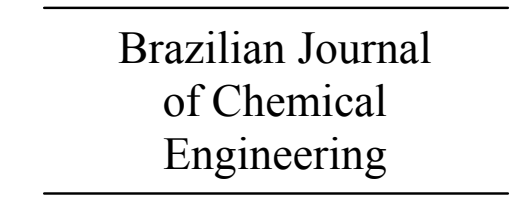

ISSN 0104-6632

Printed in Brazil

www.abeq.org.br/bjche

Vol. 30, No. 03, pp. 643 - 656, July - September, 2013

\title{
NUMERICAL ASPECTS OF DIRECT QUADRATURE-BASED MOMENT METHODS FOR SOLVING THE POPULATION BALANCE EQUATION
}

\author{
F. P. Santos ${ }^{1}$, P. L. C. Lage ${ }^{1 *}$ and C. E. Fontes ${ }^{2}$ \\ ${ }^{1}$ Programa de Engenharia Química, COPPE, Universidade Federal do Rio de Janeiro, \\ Phone: + (55) (21) 2562 8346, Fax: + (55) (21) 25628300 , \\ PO Box 68502, CEP: 21941-972, Rio de Janeiro, RJ - Brazil. \\ E-mail: paulo@peq.coppe.ufrj.br \\ ${ }^{2}$ ESSS, Av. Presidente Vargas, $3131-12^{\circ}$ floor, \\ PO Box 1203, CEP: 20210-031, Rio de Janeiro - RJ, Brazil.
}

(Submitted: April 2, 2012 ; Revised: October 2, 2012 ; Accepted: November 15, 2012)

\begin{abstract}
Direct-quadrature generalized moment based methods were analysed in terms of accuracy, computational cost and robustness for the solution of the population balance problems in the $[0, \infty)$ and $[0,1]$ domains. The minimum condition number of the coefficient matrix of their linear system of equations was obtained by global optimization. An heuristic scaling rule from the literature was also evaluated. The results indicate that the methods based on Legendre generalized moments are the most robust for the finite domain problems, while the DQMoM formulation that solves for the abscissas and weights using the heuristic scaling rule is the best for the infinite domain problems.

Keywords: Population balance; Numerical methods; DQMoM; Generalized moments; Condition number.
\end{abstract}

\section{INTRODUCTION}

There is a need to develop accurate and robust techniques for analysing the dynamics of particle systems. A proper way to obtain the evolution of the particle size distribution involves the solution of the population balance equation (PBE). The PBE is the conservation equation for the number of particles, represented by the mean number density distribution, which is a function of one or more particle properties, the internal variables, the position in the physical space, the external variables, and time (Ramkrishna, 2000).

There are several numerical methods for solving the PBE. Among them, moment based methods are been continuously developed. The method of mo- ments (MoM) (Hulburt and Katz, 1964) solves the moments of the number density distribution. Considering a mono-variate distribution $f(z, t)$ in a semi-infinite domain, $z \in[0, \infty)$, the essence of this method is to operate the PBE with the moment integral operator, $\int_{0}^{\infty} z^{k} \cdot d z, k=0,1, \ldots, \infty$, to generate differential equations for the moments of $k$-order, $\mu_{k}$. Despite being simple, this method has a closure problem because higher-order moments are usually present in the lower-order moment equations (Friedlander, 2000). McGraw (1997) proposed a methodology to solve this closure problem by approximating the integral terms in the moments equations using a $N$-point Gauss-Christoffel quadrature rule, that is, the Gaussian quadrature whose

*To whom correspondence should be addressed 
weight function is the particle number density function. This quadrature rule can be calculated from the first $2 N$ moments (Gordon, 1968; Gautschi, $1968,2004)$ and it can exactly integrate polynomials to the order $2 N-1$. Therefore, it can be used to calculate exactly the first $2 N$ moments:

$\mu_{k}=\int_{0}^{\infty} z^{k} f(z, t) d z=\sum_{\alpha=1}^{N} \varepsilon_{\alpha}^{k} \omega_{\alpha}, \quad k=0,1, \ldots$

which form a non-linear system of equations that determines the quadrature weights, $\omega_{\alpha}$, and abscissas, $\varepsilon_{\alpha}$. The method was called QMoM (Quadrature Method of Moments).

Since the integral approximations derived from the Gauss-Christoffel quadrature can also be obtained by assuming the discrete representation of the distribution function given by:

$f(z, t) \approx \sum_{\alpha=1}^{N} \omega_{\alpha}(t) \delta\left[z-\varepsilon_{\alpha}(t)\right]$

Marchisio and Fox (2005) developed the idea of solving directly for the quadrature by substituting Equation (2) into the PBE and then applying the moment integral operator. They chose to solve for the weights and weighted abscissas, $\lambda_{\alpha}=\omega_{\alpha} \varepsilon_{\alpha}$. This method, called DQMoM (Direct QMOM), does not calculate the Gauss-Christoffel quadrature in each time step, but it needs an initial discretization of the distribution function that can be given by the GaussChristoffel quadrature.

Several variants of $\mathrm{QMoM}$ and $\mathrm{DQMoM}$ have been developed. In the following, those that analysed these methods numerically are reviewed.

Alopaeus et al. (2006) proposed the usage of quadrature rules with fixed quadrature points (FixedQuadrature MoM, or FQMoM). This approach avoids some robustness and accuracy problems associated with the Gauss-Christoffel quadrature computation. By analysing several problems, they concluded that FQMoM was better than QMoM in accuracy and computational cost.

Fox (2006) applied the DQMoM to bivariate problems, including coagulation and sintering. Due to coagulation, very large particle sizes can be produced and he recommended an heuristic rule to reduce the condition number of the DQMoM system of linear equations. The condition number of the matrix of a linear system is the ratio of the largest to the smallest singular value in the singular value decomposition of this matrix. It measures the loss of accuracy in the computation of the linear system solution. If it is low, the system is said to be wellconditioned and, if it is large, the system is illconditioned. Its reduction by a linear transformation or operator can reflect positively on the stability of the DQMoM solution due to the increased accuracy of its linear system solution. This rule proposed by Fox (2006) basically scales each equation of the linear system by dividing it by an adequate power of the largest abscissa.

$\mathrm{Su}$ et al. (2007) proposed the usage of an adjustable factor, $s$, in QMoM, whose purpose is to improve the robustness in the the Gauss-Christoffel quadrature calculation. Basically, they used fractional moments given by:

$\mu_{k / s}=\int_{0}^{\infty} z^{k / s} f(z, t) d z=\sum_{\alpha=1}^{N} \varepsilon_{\alpha}^{k / s} \omega_{\alpha}$

and defined $\widetilde{z}=z^{1 / s}$ as equivalent abscissas, which were then calculated by the product-difference algorithm (Gordon, 1968).

Afterwards, Su et al. (2008) applied the same idea to DQMoM, but using an adaptive procedure to choose the value of the adjustable factor, calling the method Adaptive DQMoM (ADQMOM). The value of the adjustable factor was determined by a search procedure based on the condition number of the ADQMoM system of linear equations.

Attarakih et al. (2009) proposed the sectional QMoM (SQMoM) focusing on reconstructing the distribution function. The domain is divided into sections whose sectional moments are then used to determine a quadrature for each section, as in QMOM. Although the Gauss-Christoffel quadrature was also used, they recommended an equal-weight quadrature with better numerical properties. An interesting advantage of this method is that integral properties are not very sensitive to the reconstructed distribution.

All the direct quadrature moment methods described above used the monomial moment integral operator and the formulation based on the weights and weighted abscissas.

On the other hand, Grosch et al. (2006) presented a generalized framework for the quadrature method of moments that is based on the concepts of generalized moments and coordinate transformations. In analogy to the QMoM, this framework transforms the PBE using the generalized moment integral operator, $\int_{0}^{\infty} \phi_{k}(z) \cdot d z$, where $\phi_{k}(z)$ may be an orthogonal polynomial (QMoGeM). They formulated the QMOM equations as a differential algebraic 
system of equations (QMoM-DAE) by solving the QMoM moment equations simultaneously with:

$\mu_{k}^{\phi}=\int_{0}^{\infty} \phi_{k}(z) f(z, t) d z=\sum_{\alpha=1}^{N} \phi_{k}\left(\varepsilon_{\alpha}\right) \omega_{\alpha}$

They also derived a method by applying an index reduction procedure to $\mathrm{QMoM}-\mathrm{DAE}$, which produced the DQMoM in terms of weights and abscissas when the monomial polynomial basis was used, which is called here DQMoMa. The framework developed by Grosch et al. (2006) also includes the DQMoM developed by Marchisio and Fox (2005), the QMoM developed by McGraw (1997) and their variations with generalized moments (DQMoGeM). Grosch et al. (2006) tested these variants of QMoM by comparing their solutions of mono-variate PBE problems in the semi-infinite domain to those obtained by the Parsival solver (Wulkow et al., 2001), which ensures a specified tolerance by using a hpadaptive discontinuous finite elements method. Grosch et al. (2006) concluded that the methods based on Laguerre generalized moments were only marginally more robust than the corresponding methods using the monomial moments. They recommended the usage of the DQMoM formulation derived using the monomial moment integral operator and solved in terms of the quadrature weights and abscissas because of its good robustness and low computational cost.

Although Grosch et al. (2006) did analyse the numerical behavior of quadrature moment methods using generalized moments, the analysis was limited to Laguerre generalized moments due to the choice of population balance problems in semi-infinite domains. However, there is an increasing interest in moment methods for problems in finite domains (Strumendo and Arastoopour, 2008, Lage, 2011). Therefore, the main goal of the present work is to analyse the numerical properties of direct quadrature moment methods using generalized moments both in semi-infinite and finite domains with Laguerre and Legendre polynomials, respectively. The formulations that solve either for the abscissas or for the weighted abscissas were also compared.

\section{POPULATION BALANCE MODELING}

Consider the population balance equation with an unique additive internal variable, $z$, represented by the following form of the PBE:

$$
\begin{aligned}
& \frac{d f(z, t)}{d t}=v \int_{z}^{z} \max ^{\max } b(z) P\left(z \mid z^{\prime}\right) f\left(z^{\prime}, t\right) d z^{\prime}-b(z) f(z, t) \\
& +\frac{1}{2} \int_{0}^{z} a\left(z-z^{\prime}, z^{\prime}\right) f\left(z-z^{\prime}, t\right) f\left(z^{\prime}, t\right) d z^{\prime} \\
& -\int_{0}^{z \max } a\left(z, z^{\prime}\right) f(z, t) f\left(z^{\prime}, t\right) d z^{\prime}+R(z, t)
\end{aligned}
$$

where $a\left(z, z^{\prime}\right)$ is the aggregation frequency of the particles with property $z$ and $z^{\prime}, b(z)$ is the breakage frequency of a particle with the property $z$ and $P\left(z \mid z^{\prime}\right)$ is the daughter probability distribution function for the breakage of a particle with property $z^{\prime}, v$ is the mean number of particles formed in the breakage process and $R$ is an additional source term, which can represent, for instance, nucleation or growth. The first and the second terms are, respectively, the birth and death terms for breakage, and the third and the fourth are the corresponding terms for aggregation.

\section{DIRECT QUADRATURE METHODS}

Following Marchisio and Fox (2005), the DQMoGeM is derived by substituting Equation (2) into the PBE and then applying the generalized moment integral operator. It is then possible to define the following formulations.

\section{Formulation Using Weights and Weighted-Abscissas (DQMoGeM)}

$\frac{d \omega_{\alpha}}{d t}=\theta_{\alpha}, \quad \frac{d \lambda_{\alpha}}{d t}=\rho_{\alpha}, \quad \alpha=1, \cdots, N$

which must be solved together with the following system of linear equations:

$$
\begin{aligned}
& \sum_{\alpha=1}^{N}\left(\phi_{k}\left(\varepsilon_{\alpha}\right)-\varepsilon_{\alpha} \phi_{k}{ }^{\prime}\left(\varepsilon_{\alpha}\right)\right) \theta_{\alpha}+\sum_{\alpha=1}^{N} \phi_{k}{ }^{\prime}\left(\varepsilon_{\alpha}\right) \rho_{\alpha} \\
& =S_{k}, \quad k=0, \cdots, 2 N-1
\end{aligned}
$$

where $S_{k}$ is defined below. If $\phi_{k}(z)=z^{k}$, the DQMoGeM is reduced to the standard form of DQMoM. Fox's (2006) heuristic rule consists of dividing the $k$ equation of Equation (7) by $\varepsilon_{\max }^{k}$, where $\varepsilon_{\max }=\max _{\forall j} \varepsilon_{j}$. 
Formulation Using Weights and Abscissas (DQMoGeMa)

$\frac{d \omega_{\alpha}}{d t}=\theta_{\alpha}, \quad \frac{d \varepsilon_{\alpha}}{d t}=v_{\alpha}, \quad \alpha=1, \cdots, N$

which must be solved together with the following system of linear equations:

$\sum_{\alpha=1}^{N} \phi_{k}\left(\varepsilon_{\alpha}\right) \theta_{\alpha}+\sum_{\alpha=1}^{N} \omega_{\alpha} \phi_{k}{ }^{\prime}\left(\varepsilon_{\alpha}\right) v_{\alpha}=S_{k}, \quad k=0, \cdots, 2 N-1$

If $\phi_{k}(z)=z^{k}$, the DQMoGeMa is reduced to what is called here as the DQMoMa.

\section{Source Terms Due to Aggregation and Breakage}

Using the Gauss-Christoffel quadrature approximation, the sources terms in the system of linear equations are given by:

$$
\begin{aligned}
S_{k}(t) & =\int_{0}^{z_{\max }} \phi_{k}(z) S(z, t) d z \\
& =B_{k}^{a}(t)-D_{k}^{a}(t)+B_{k}^{b}(t)-D_{k}^{b}(t)+R_{k}(t)
\end{aligned}
$$

where:

$$
\begin{aligned}
& B_{k}^{a}(t)=\int_{D} B^{a}(z, t) \phi(z) d z \\
&=\frac{1}{2} \sum_{\beta=1}^{N} \sum_{\alpha=1}^{N} \phi_{k}\left(\varepsilon_{\alpha}+\varepsilon_{\beta}\right) a_{\alpha \beta} \omega_{\alpha} \omega_{\beta} \\
& D_{k}^{a}(t)=\int_{D} D^{a}(z, t) \phi(z) d z=\sum_{\beta=1}^{N} \sum_{\alpha=1}^{N} \phi_{k}\left(\varepsilon_{\alpha}\right) a_{\alpha \beta} \omega_{\alpha} \omega_{\beta} \\
& B_{k}^{b}(t)=\int_{D} B^{b}(z, t) \phi(z) d z=\sum_{\alpha=1}^{N} b_{\alpha} \phi_{k}\left(\varepsilon_{\alpha}\right) \omega_{\alpha} \\
& D_{k}^{b}(t)=\int_{D} D^{b}(z, t) \phi(z) d z=\sum_{\alpha=1}^{N} b_{\alpha} \nu_{\alpha} \omega_{\alpha} \Pi_{\alpha}^{k} \\
& R_{k}(t)=\int_{D} R(z, t) \phi(z) d z \\
& \Pi_{\alpha}^{k}=\int_{0}^{\varepsilon_{\alpha}} \phi_{k}(z) P\left(z \mid \varepsilon_{\alpha}\right) d z, \quad \alpha=1, \cdots, N
\end{aligned}
$$

If $\phi_{k}(z)=z^{k}$ these terms become those of DQMoM and QMoM (Marchisio and Fox, 2005). It is important to point out that Equations (15) and (16) are calculated analytically or by an adaptive quadrature with error control.

\section{NUMERICAL PROCEDURE}

The implementation of the PBE solution was performed in $\mathrm{C}$ and FORTRAN. The source codes were compiled with gfortran and g++ using the -03 high optimization flag with double precision variables. The work was carried out on an Intel(R) Core(TM)2 Quad CPU Q6600 2.40GHz processor with Ubuntu 10.04 Linux operating system.

The integration of Equations (15) and (16) was performed using the adaptive quadrature AUTOQUAD routine (Lage, 1992). The ORTHOPOL package (Gautschi, 1994) was used to calculate the recursion coefficients of the orthogonal polynomials. For the initial conditions, the modified Chebyshev algorithm was used to obtain the Gauss-Christoffel weights and abscissas from the initial values of the generalized moments (Gautschi, 1994).

The time integration was performed using the DASSLC package (Secchi, 2007), an adaptive time step and variable order integrator based on backward differentiation formulas, with required relative and absolute tolerances equal to $10^{-10}$. This made the time integration error much smaller than the quadrature error embodied in these quadrature moment methods.

An evaluation of the computational time for all methods was performed for 1.0 second of simulation. The clock intrinsic subroutine of $\mathrm{g}++$ was used to obtain the elapsed $C P U$ time in seconds with $0.01 \mathrm{~s}$ of accuracy.

The computation of the condition number of the coefficient matrix, A, of the system of linear equations given by Equation (7) or Equation (9) was carried out by DGTRF and DGECON routines of the LAPACK package (Anderson et al., 1999).

In order to evaluate whether the robustness of the solution of the linear system of equations could be improved, a diagonal pre-conditioner, $\mathbf{P}=\operatorname{diag}(\mathbf{p})$ was applied to the linear system given by Equation (7) or (9) and the minimum condition number (Marechal and Ye, 2009) of the resulting coefficient matrix was obtained by defining the following minimization problem:

$$
C N_{\min }=\min _{\mathbf{p} \in \Re^{2 N}}[C N(\mathbf{P A})]
$$

This minimization was performed with the 
NLOPT package (Johnson, 2008). The results obtained for the minimum condition number of DQMoM were compared against the condition number obtained using the heuristic rule proposed by Fox (2006).

\section{RESULTS AND DISCUSSION}

The methods presented in section "Direct Quadrature Methods" were applied to four test cases for which analytical solutions are known using the two formulations derived with the monomial (DQMoM and DQMoMa) and generalized moment (DQMoGeM and DQMoGeMa) integral operators.

The first two test cases used the population balance problem in a semi-infinite domain described in Equation (7) and the solution can use the monic Laguerre generalized moments. Case I is aggregation dominant with $\Phi(\infty)=0.5$ and case II is breakage dominant with $\Phi(\infty)=2$. Both cases were solved up to $t=1$, where steady-state is basically reached. The last two test cases are for a pure breakage problem in a finite domain as given in Equation (8) and they can be solved using monic shifted Legendre generalized moments. Case III defines $\gamma=2$ and it is easier to solve than case IV with $\gamma=1 / 3$. Cases III and IV were solved up to $t=5$.

These problems are sufficiently simple to allow all methods to converge for relatively small $N$ values. In other words, if a relatively small $N$ is used, the quadrature errors are not too large to generate two abscissas close to each other during integration. This is a well-known problem that would render the matrix of the system of linear equations singular, which would make the method comparison difficult. It should be pointed out that the breakage and aggregation kernels do not affect directly the coefficient matrix of the linear system of equations in these methods.

\section{Convergence Analysis}

Since the analytical solutions are known, the relative error of each regular moment can always be calculated by:

$X_{k}=\left|\frac{\mu_{k}^{(e)}-\mu_{k}}{\mu_{k}^{(e)}}\right|$

where $\mu_{k}^{(e)}$ are the analytical moments and $\mu_{k}$ are the moments reconstructed from the numerical solution using Equation (1). Since the first six moments are used in the solution for $N \geq 3$, the convergence of the quadrature moment methods was evaluated using their mean square relative error:

$$
X_{R M S, 6}=\sqrt{\frac{1}{6} \sum_{k=0}^{5} X_{k}^{2}}
$$

The regular moments were used to evaluate the convergence even for the methods that use generalized moments.

Figure 1 shows the time evolution of $X_{R M S, 6}$ for cases I, II, III and IV solved with $N=5$. All cases show initial $X_{R M S, 6}$ values of the order of the required integration tolerances, but they increase during the integration due to the accumulation of quadrature error along the time integration. Nevertheless, at the end of the simulations, although the final $X_{R M S, 6}$ values vary from case to case, the errors are still acceptable. As expected, the largest error is for case IV, due to the fact that the breakage frequency in this case does not belong to the space of polynomials, which increases the quadrature error.

The $X_{R M S, 6}$ values in the solution of all cases by all methods are compared in Figure 2 for several $N$ values at the end of the simulations. Two kinds of convergence behavior can be seen in Figure 2 that are exemplified for the simulations of case III. The first behavior can be seen in the DQMoGeM and DQMoGeMa simulations, which converge with an almost constant rate up to a $X_{R M S, 6}$ value that is about 1-2 orders of magnitude larger than the tolerance in the time integration used in DASSLC. Then, for large $N$ values, the $X_{R M S, 6}$ value remains almost constant, indicating that the accumulated time integration error becomes more important than the quadrature error. The second kind of behavior can be seen in the DQMoM and DQMoMa simulations for case III, which converges with an almost constant rate up a $N$ value where the convergence rate decreases and, sometimes, the $X_{R M S, 6}$ value even increases. For even larger $N$ values, the solution could not be completed because the DASSLC routine could not perform the integration with the required local accuracy due to the numerical error in the solution of the linear system of the moment method. In other words, both behaviors can be explained by the following reasoning. If the linear system of the direct quadrature moment method is well-conditioned, the convergence rate is almost constant until $N$ increases up to a point when the time integration error became larger than the 


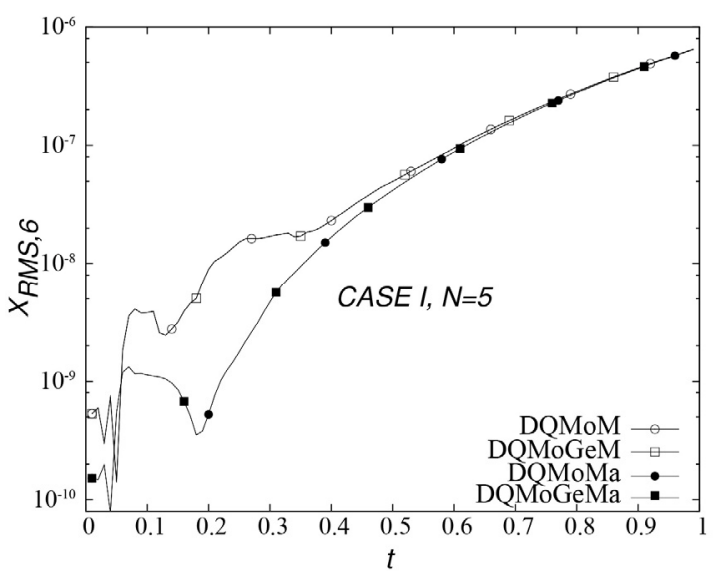

(a)

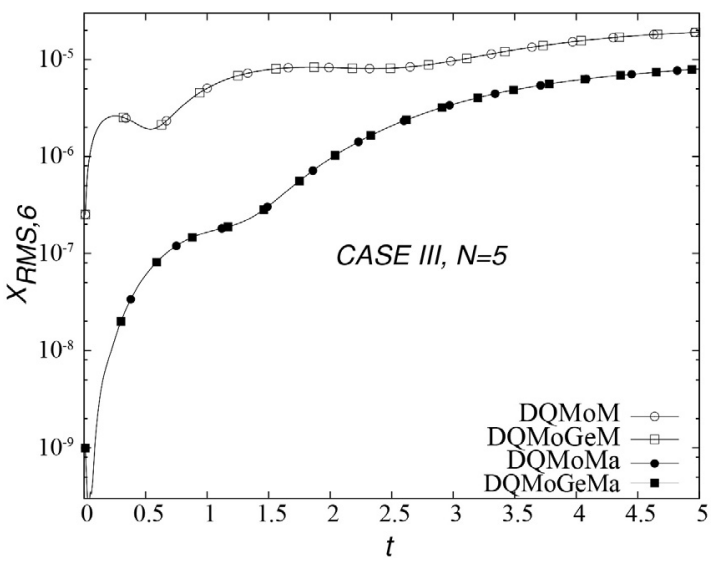

(c)

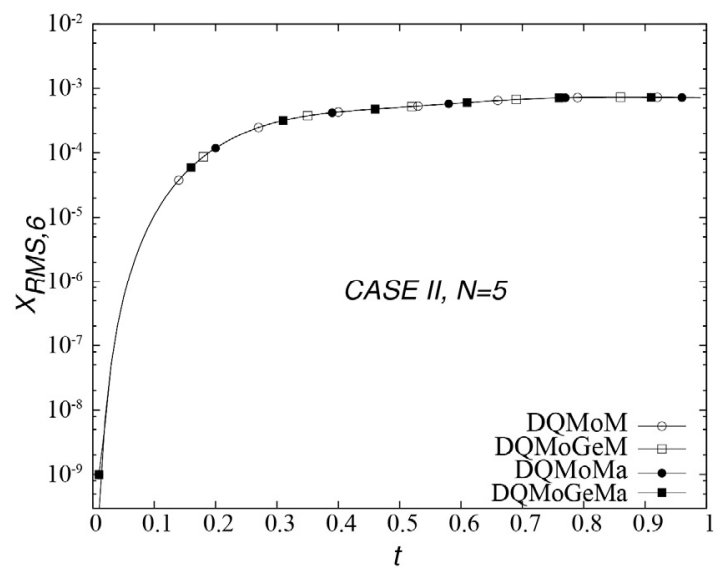

(b)

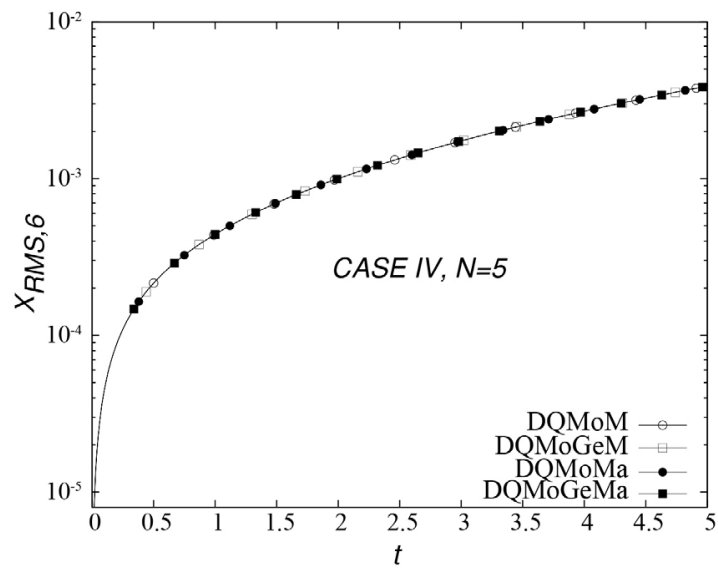

(d)

Figure 1: Behavior of $X_{R M S, 6}$ during simulation using $N=5$ for: (a) case I, (b) case II, (c) case III and (d) case IV.

quadrature error, when $X_{R M S, 6}$ becomes almost constant. However, if the linear system becomes illconditioned, the numerical error deteriorates the convergence or even makes the DASSLC routine give up trying to perform the integration. This lack of robustness caused by the numerical error in the linear system solution is analysed in section "Robustness Analysis".

\section{Computational Cost}

Figure 3 shows the computational time for several values of $N$ for all methods and for all test cases. Again, two behaviors can be perceived and they are again exemplified for case III. The first one can be seen in the DQMoGeMa solution, for which the computational time of the simulation steadily increases with $N$. The second behavior occurs for all other methods for case III, but the DQMoM and DQMoMa simulations are better examples. In this behavior, the computational time versus $N$ value curve suffers a strong slope change at some $N$ value. Again, this abnormal change of slope is related to the loss of robustness, which makes the DASSLC routine spend much more time to achieve the required tolerance.

It is clear from Figure 3 that, before losing robustness, DQMoM and DQMoMa are faster than the generalized moment methods. In infinite domain problems, they are 10 times faster but, for the finite domain problems, the generalized moment methods are only 3-4 times slower. 


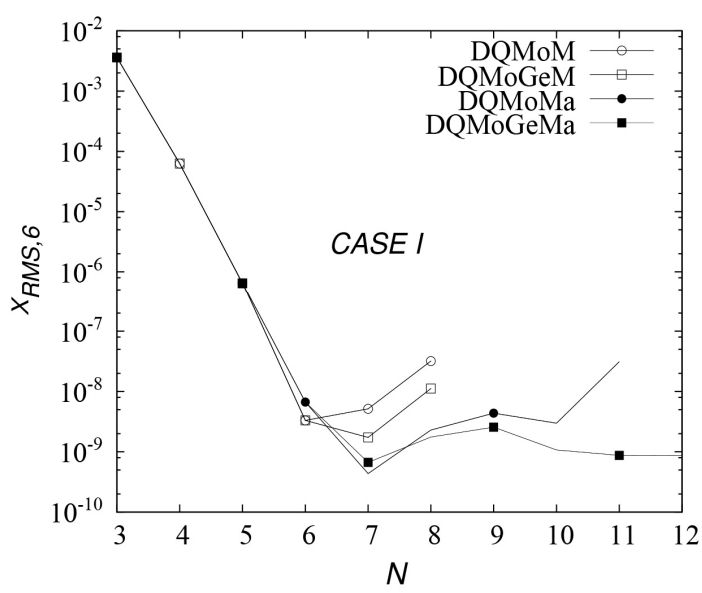

(a)

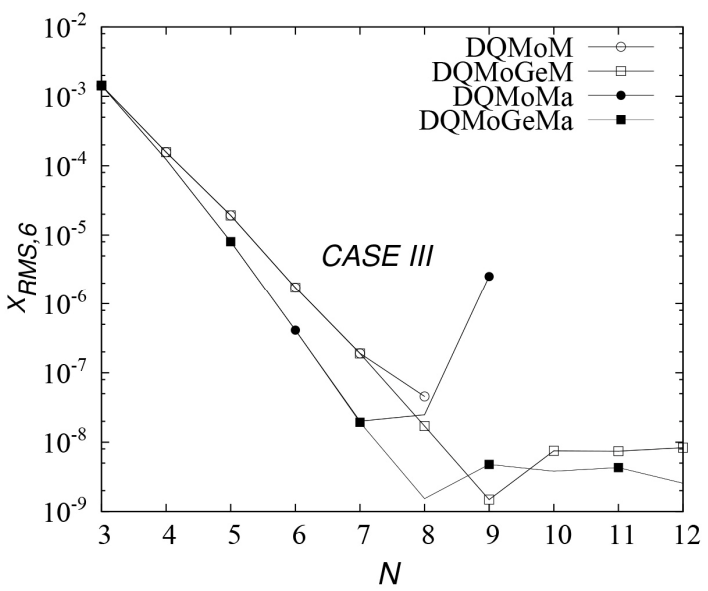

(c)

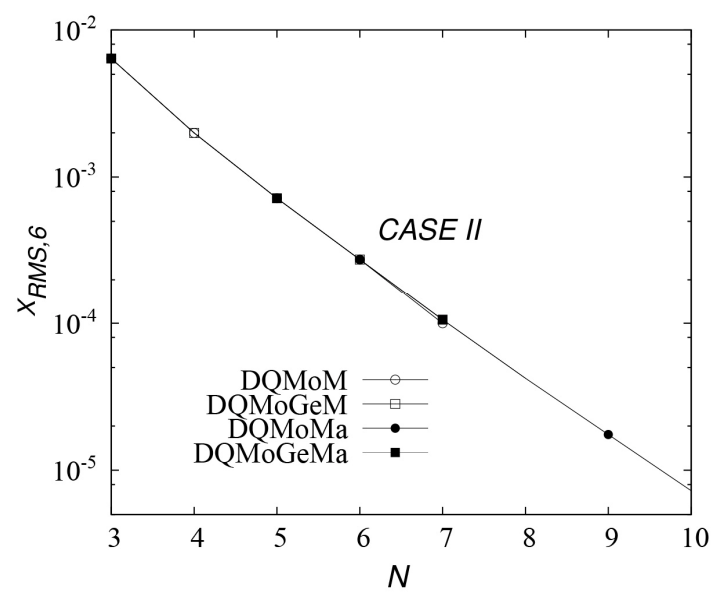

(b)

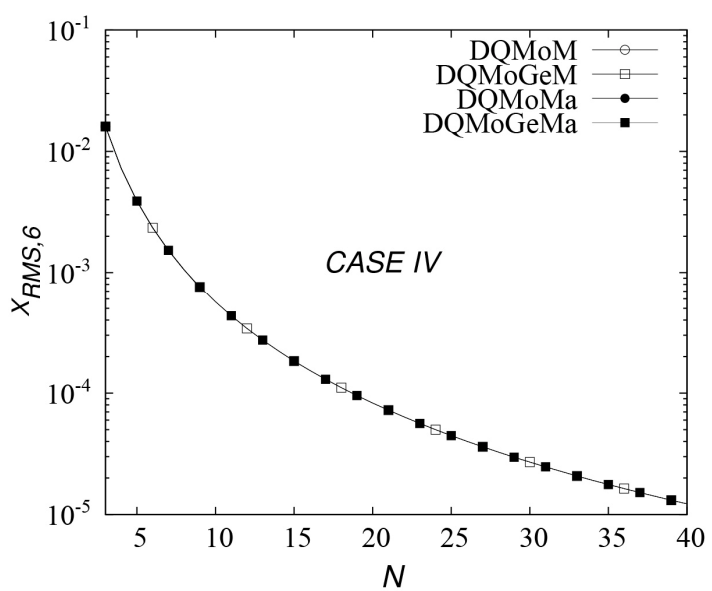

(d)

Figure 2: Values of $X_{R M S, 6}$ at the end of the simulation using several $N$ values for: (a) case I, (b) case II, (c) case III and (d) case IV.

\section{Robustness Analysis}

It is usually considered that the loss of robustness in these methods comes from the numerical error embodied in the solution of the linear system of equations, Equations (7) and (9), as its matrix becomes singular. For some problems, this happens when two abscissas become very close to each other due to error accumulation during the solution. For this case, there are some solutions. One of these is the usage of a fractional moment operator with an adapted parameter to create a different linear system with a better condition number (Su et al., 2008). The other solution is to keep the same moment integral operator and scale the equations (Fox, 2006). Here we analysed this last solution in a systematic way.

The condition number of the coefficient matrix of the unscaled linear system of equations, Equations (7) and (9), was evaluated for each simulation that could be integrated up to its final time value and exactly at this instant. The minimum condition number using a diagonal pre-conditioner was also obtained at this time to verify if the solution could be improved by scaling.

Figure 4 shows the condition number obtained for all test cases and methods without any scaling and for different $N$ values. Regarding the two formulations of the methods, this figure clearly shows that there is no significant difference in the condition number values for both formulations of the same method. For case I, there is not much difference between the condition numbers for all methods. On the other hand, for all other cases, especially for the finite domain problems, the generalized moment methods have smaller condition numbers than those for the monomial moment methods. For case I, although the values of the condition numbers are close for all methods, DQMoMa has shown to be 


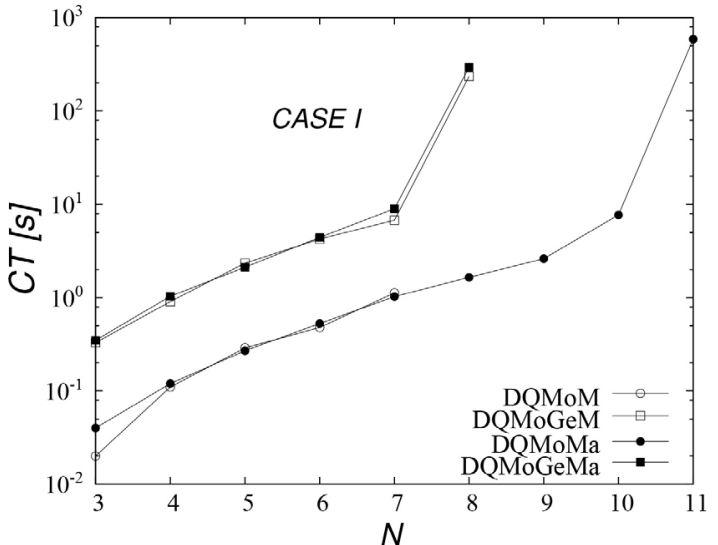

(a)

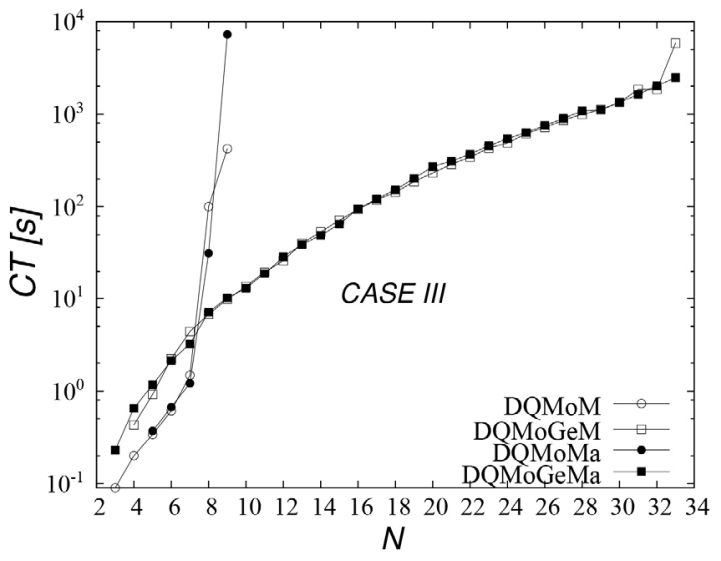

(c)

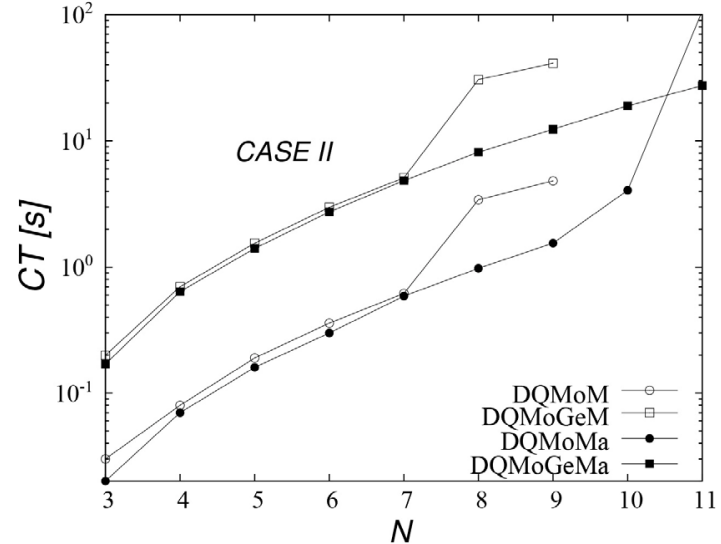

(b)

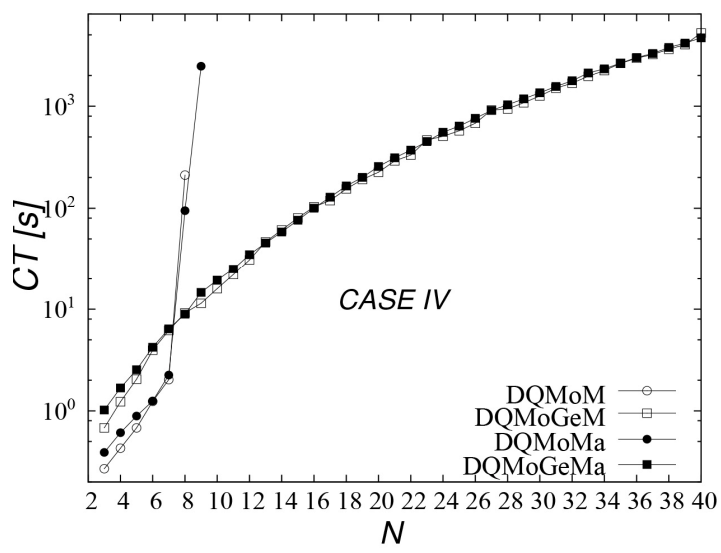

(d)

Figure 3: Computational time using several $N$ values for: (a) case I, (b) case II, (c) case III and (d) case IV.

more robust than the other methods. For case II, it was as robust as DQMoGeMa, although its condition number is $10^{8}$ times larger than that for DQMoGeMa at $N=11$. This behavior was also found by Grosch et al. (2006), who compared methods using monomial and Laguerre generalized moments. For the finite domain problems, the condition number values are $10^{2}-10^{3}$ times smaller for the Legendre generalized moment methods. This fact is reflected in the method robustness, as cases III and IV could be solved by DQMoGeM and DQMoGeMa using $N$ values above 30, while DQMoM and DQMoMa could not be employed above 10 quadrature points. This was not observed by Grosch et al. (2006) because they did not solve problems in finite domains.

In order to verify the possible robustness gain by the scaling of the coefficient matrix, all test cases were solved by all methods using a diagonal preconditioner obtained at time zero by an optimization procedure. Figure 5 shows the ratio between the minimum condition number and the condition number for all test cases and methods and for different $N$ values at the end of the simulations. For the problems in a semi-infinite domain, the reduction of the matrix condition number by scaling increases almost exponentially, and a $10^{10}-10^{15}$ reduction is achieved for $N=10$. For the finite domain problems, the condition number was only sligthly affected by the optimization for DQMoM and DQMoMa. However, the values of the minimum condition number were much smaller than the original $C N$ values for both DQMoGeM and DQMoGeMa, which are related to the use of the Legendre polynomial moments. The ratio between the minimum and the original condition numbers drops very fast with $N$. The robustness gain was large, as the maximum $N$ value in the solution of cases III and IV increased from around 30 to above 50. Therefore, the optimization procedure applied at $t=0$ was a very effective way of improving the robustness of the quadrature methods based on Legendre polynomial moments applied to finite domain problems. 


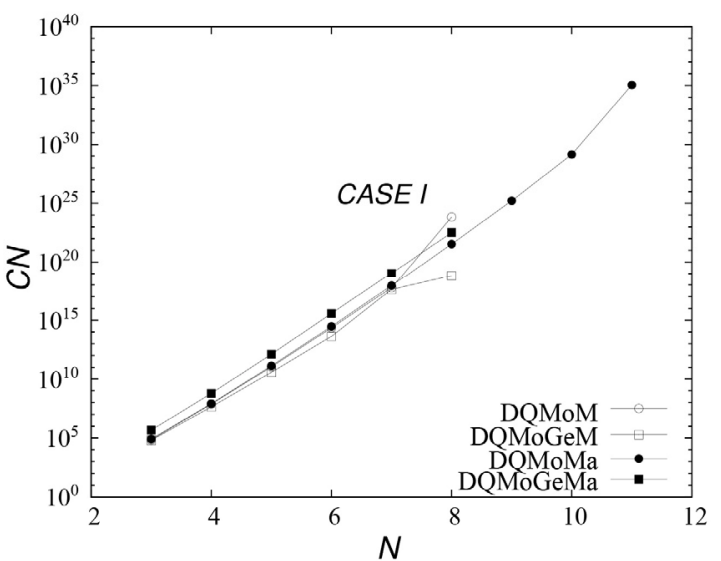

(a)

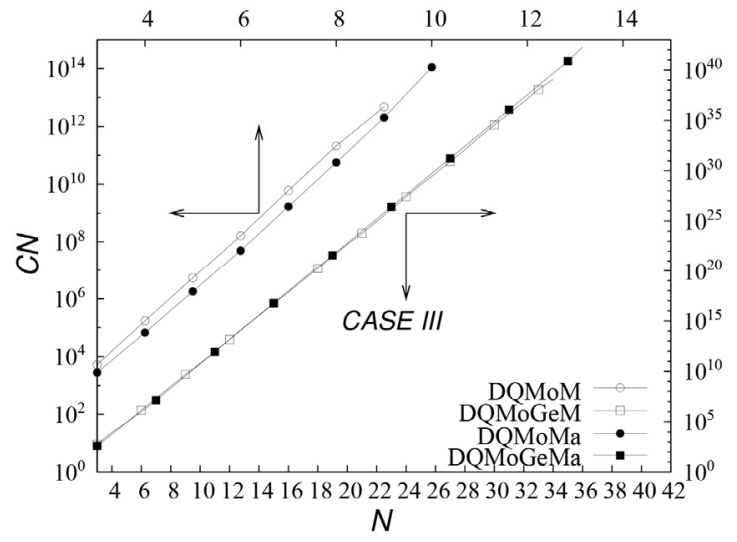

(c)

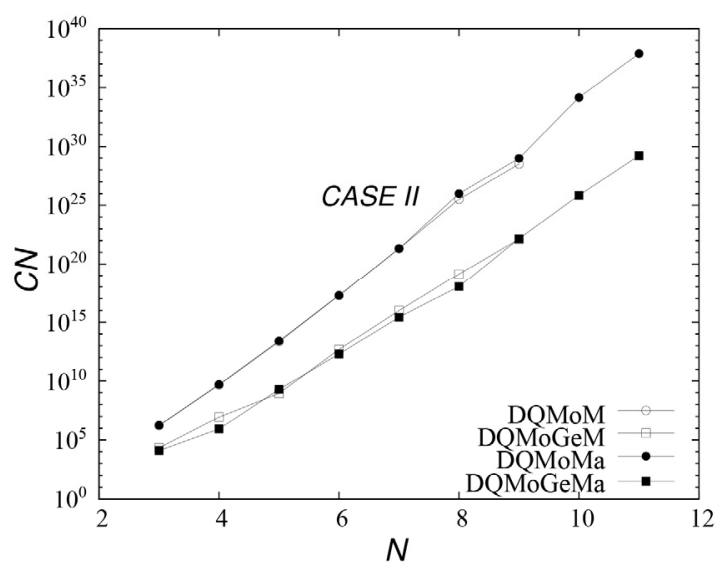

(b)

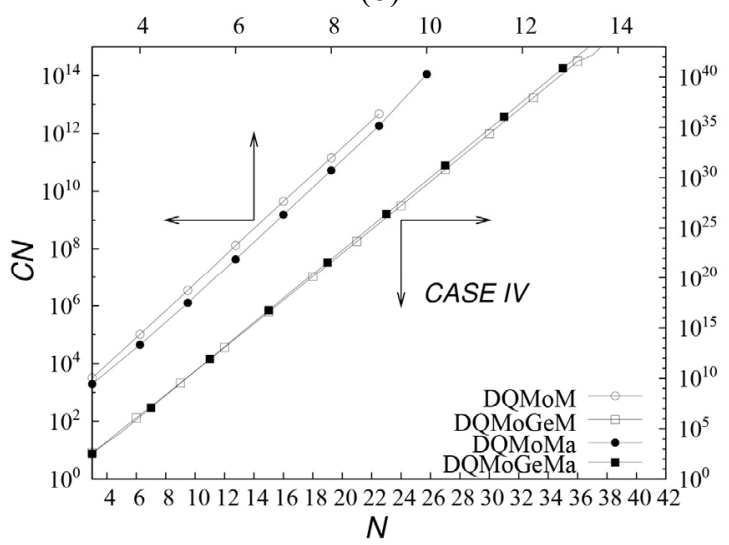

(d)

Figure 4: Condition number of the coefficient matrix of all methods using several $N$ values for: (a) case I, (b) case II, (c) case III and (d) case IV.

A sensitivity analysis was carried out to verify if the $C N_{\min } / C N$ behavior depends on the breakage kernel parameters. The parameters $\Phi(\infty)$ and $\gamma$ of cases I and III were modified to $\Phi(\infty)=0.1$ and $\gamma=3$, generating cases $\mathrm{V}$ and $\mathrm{VI}$, respectively. The corresponding results for $\mathrm{CN}_{\min } / \mathrm{CN}$ are shown in Figure 6. It can be seen that these perturbations in the parameters do not change the $C N_{\min } / C N$ behavior observed in Figures 5(a) and 5(c). Besides, the robustness improvement was the same. It seems to be independent of the dynamics of the problem.

The heuristic rule of Fox (2006) was extended for all methods and for cases I and III. Figure 7 shows the ratio between the minimum condition number and the condition number calculated employing this heuristic rule evaluated at the end of the simulations, using all methods and for different $N$ values.

For case I, Figure 6 shows that the heuristic rule proposed by Fox (2006) reduces significantly the condition number of the original linear system of equation of DQMoM and DQMoMa since the $C N_{\min } / C N_{F o x}$ value is almost always between 0.1 and 1 for these methods. The rule also worked reasonably well for the Laguerre generalized moment methods $\left(10^{-5} \lesssim C N_{\text {min }} / C N_{F o x} \lesssim 0.3\right)$, probably because monic polynomials were used and the semi-infinite domain generates large abscissa values that make the largest order polynomial term much larger than the others. In these problems in semi-finite domains, the robustness gain obtained was not too impressive, as both scaling rules allowed us to obtain the solution for only one more quadrature point for case I solved by DQMoM and DQMoMa.

As can be seen in Figure 6, Fox's rule did not work at all for case III using Legendre polynomial moments. The behavior of $C N_{\min } / C N_{F o x}$ for case III was basically equal to that of $C N_{\min } / C N$ shown in 


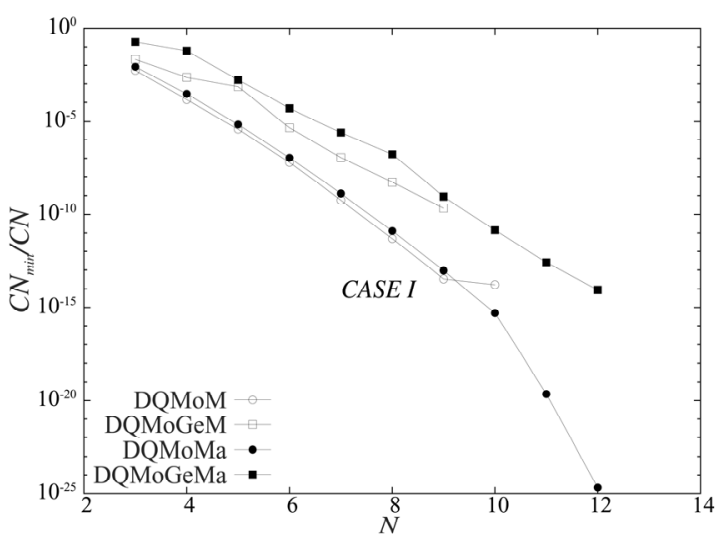

(a)

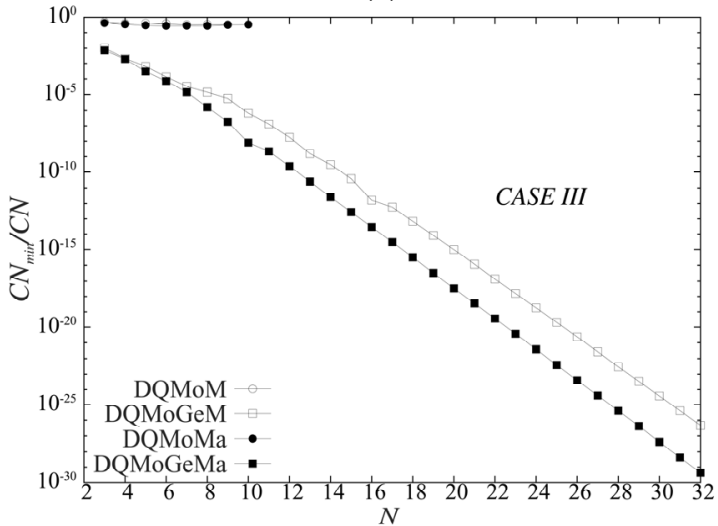

(c)

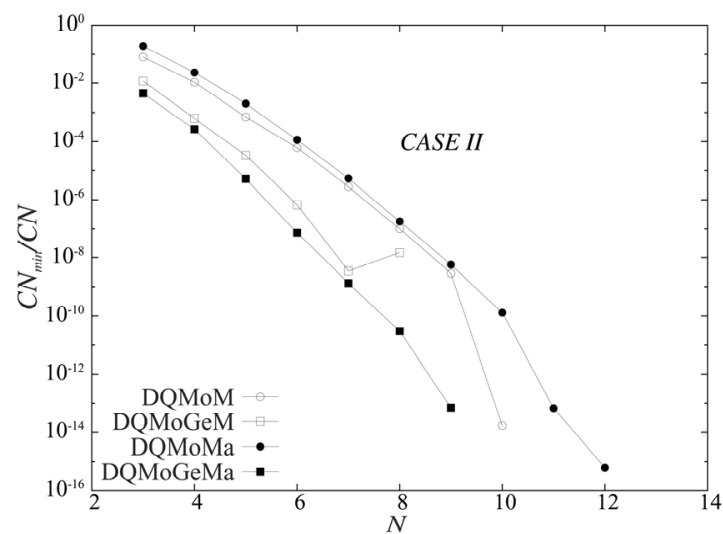

(b)

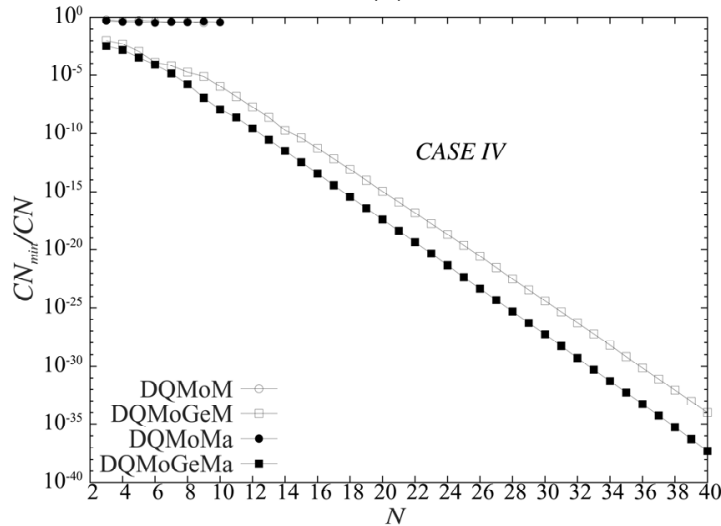

(d)

Figure 5: Reduction of the condition number of the coefficient matrix of all methods by minimization using several $N$ values for: (a) case I, (b) case II, (c) case III and (d) case IV.

Figure 5. This was expected as the largest abscissa is not related to the value of $\phi_{k}\left(\varepsilon_{\alpha}\right)$ for the Legendre polynomials in the $[0,1]$ domain. Besides, no robustness gain was obtained for the finite domain problems for all methods using Fox's heuristic

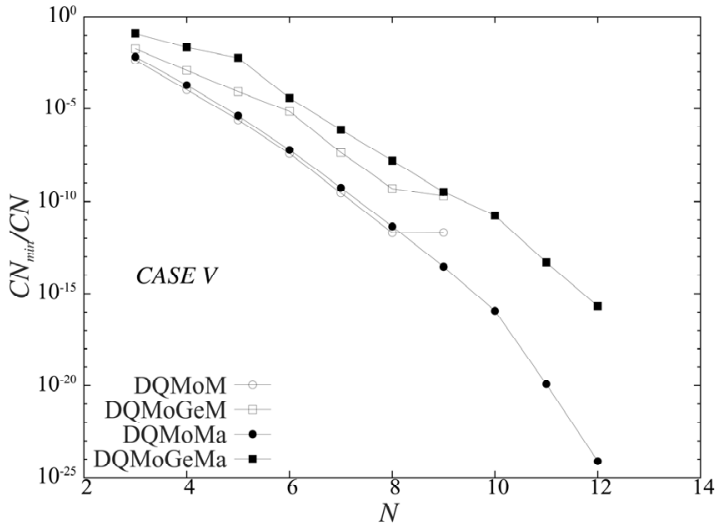

(a) scaling rule. On the other hand, as commented above, the optimization procedure applied to this case increased the robustnesses of the DQMoGeM and DQMoGeMa solutions. This behavior was also observed for cases $\mathrm{V}$ and VI.

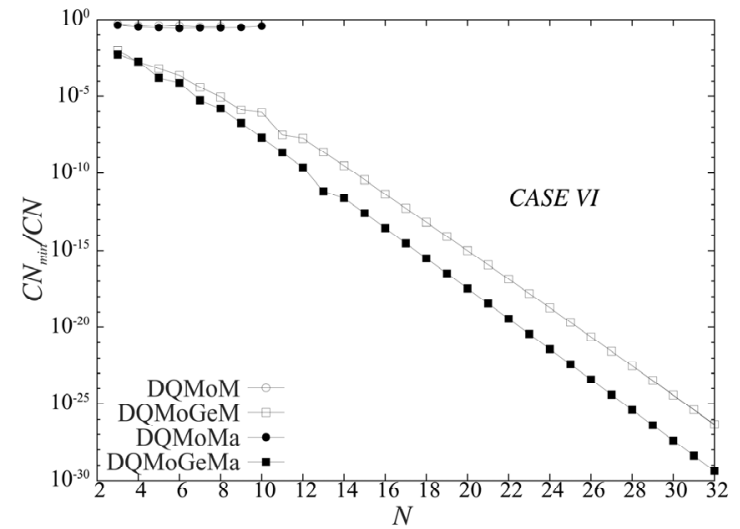

(b)

Figure 6: Reduction of the condition number of the coefficient matrix of all methods by minimization using several $N$ values for: (a) case V and (b) case VI (sensitivite analysis). 


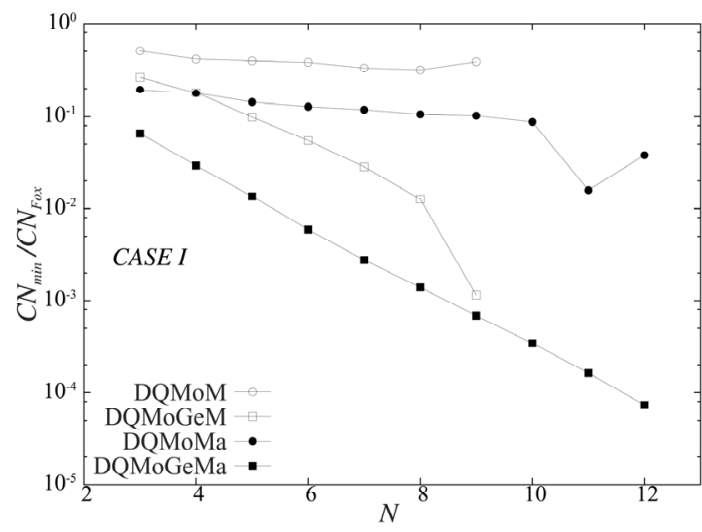

(a)

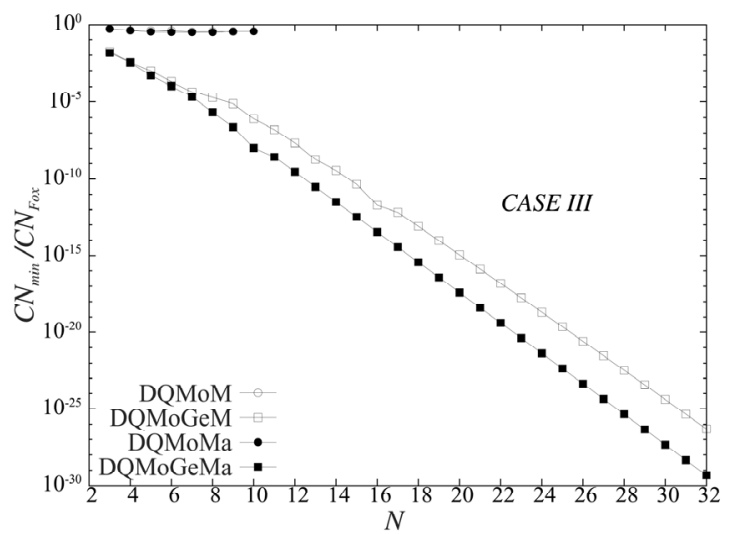

(b)

Figure 7: Reduction of the condition number of the coefficient matrix of all methods by minimization using several $N$ values for: (a) case I and (b) case III.

Figure 8 shows the ratio between the computational time of the simulations using the minimum condition number and Fox's heuristic scaling rule for cases I and III and for several $N$ values. This ratio decreases with $N$ because the simulation time increases faster with $N$ than the optimization time. For case I, both methods have the same robustness but Fox's heuristic scaling rule is computationally much cheaper. This is expected to happen for all semi-infinite domain problems for which the computational cost of the optimization procedure used to calculate $C N_{\min }$ at $t=0$ is a large fraction of the total computational time. For case III, the

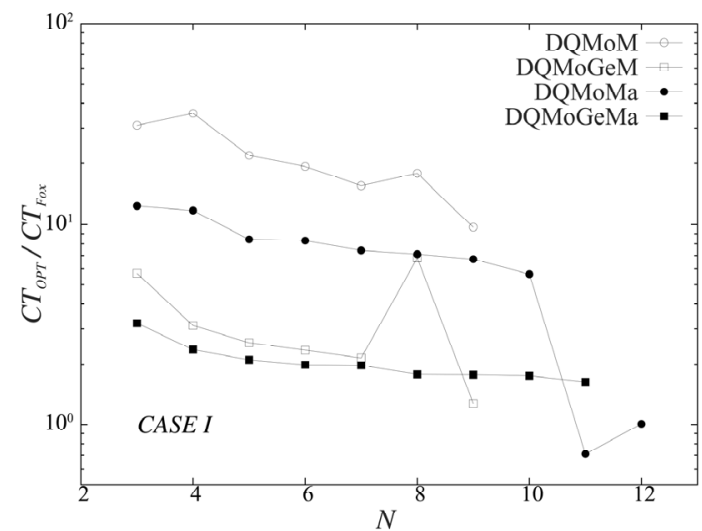

(a) scaling using the optimization procedure for $C N_{\min }$ determination is more robust than Fox's heuristic scaling rule and Figure 7 shows that its additional computational cost becomes less important as $N$ increases. The strong slope change in the curves for DQMoM and DQMoMa are related to the loss of robustness, which makes the simulation time become larger than the optimization time. Therefore, for Legendre moment methods applied to problems in finite domains, it is expected that the computation of $C N_{\min }$ is justified due to the increased robustness, especially when the simulation time is quite large, as in PB-CFD simulations.

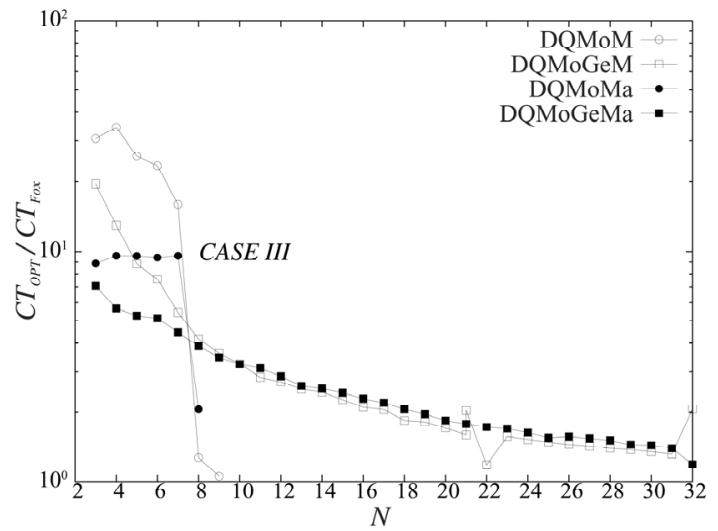

(b)

Figure 8: Ratio between the computational time of the simulations using the minimum condition number and the condition number obtained by Fox's (2006) rule for all methods using several $N$ values for: (a) case I and (b) case III. 


\section{CONCLUSIONS}

Direct quadrature methods based on regular and orthogonal polynomial moments were analysed using the weighted-abscissa and abscissa formulations by solving population balance problems in semi-infinite and finite domains. The methods were compared regarding their accuracy, computational cost and robustness.

The methods based on regular moments were always the fastest. However, they were the least robust for the finite domain test cases. For these problems, the Legendre generalized moment methods were the most robust. The DQMoM formulation based on the abscissas (DQMoMa) proved to be more robust than the weighted-abscissa formulation (DQMoM) for the problems in the semiinfinite domain.

The best scaling of the linear system of equations of all methods was obtained by global minimization of the condition number of the transformed coefficient matrix using a diagonal preconditioning matrix. The results showed only a small improvement over Fox's heuristic rule for the methods based on regular moments applied to problems in the semi-infinite domain. In this case, both scaling methods allowed the PBE to be solved with only one extra quadrature point. For the methods based on regular moments, the optimization procedure did not give a robustness gain when applied to problems in the finite domain. On the other hand, for the methods based on Legendre polynomial moments, the scaling by optimization yielded a large reduction in the condition number, allowing the solution for a much larger number of quadrature points.

The heuristic rule of Fox (2006) had almost the same performance as the scaling obtained by optimization for the problems in the semi-infinite domain, but with less computational cost. However, for problems in the finite domain, the scaling by minimizing the condition number was much better, inproving the method robustness. A sensitive analysis has shown that the results seem to be independent of the problem dynamics.

Therefore, it can be concluded that, for problems in the semi-infinite domain, DQMoMa should be used with Fox's (2006) scaling rule. This conclusion agrees with Grosch et al. (2006), who found that DQMoMa is better than other QMoM variations studied by them for semi-finite domain problems. However, for problems in finite domains, the generalized Legendre moment methods should be used with the scaling obtained by the minimization of the coefficient matrix condition number, especially if a large number of quadrature points is required or when the simulation time is large.

\section{ACKNOWLEDGMENTS}

Paulo L. C. Lage and Fabio P. Santos acknowledge the financial support of $\mathrm{CNPq}$, grants nos. 302963/2011-1, 478589/2011-5 and 140794/2010-7.

\section{NOMENCLATURE}

$\begin{array}{ll}\text { A } & \text { coefficient matrix } \\ a & \text { aggregation frequency } \\ B & \text { birth term } \\ b & \text { breakage frequency } \\ C N & \begin{array}{l}\text { condition number } \\ \text { computational time in }\end{array} \\ C T & \begin{array}{l}\text { seconds } \\ \text { death term }\end{array} \\ D & \text { number density distribution } \\ f & \text { function } \\ H & \text { Heaviside step function } \\ N & \text { number of quadrature points } \\ P & \text { probability density function } \\ & \begin{array}{l}\text { of breakage of particles } \\ \text { preconditioning matrix }\end{array} \\ \mathbf{P} & \text { pre-conditioner diagonal } \\ & \text { elements } \\ R & \text { source term } \\ S & \text { adjustable factor } \\ S_{k} & \text { moment of } k \text { order of } \\ & \text { source term } \\ t & \text { time } \\ X & \text { relative error } \\ z & \text { additive internal variable }\end{array}$

\section{Greek Letters}

$\begin{array}{ll}\gamma & \begin{array}{l}\text { breakage kernel parameter } \\ \text { in the finite domain problem }\end{array} \\ \delta & \text { Dirac delta function } \\ \varepsilon & \text { abscissa } \\ \lambda & \text { weighted abscissa } \\ \mu_{k} & \text { moment of order } k \\ v & \text { number of particles formed } \\ & \text { in breakage } \\ \omega & \text { weight function } \\ \Phi & \text { the ratio } \mu_{0}(t) / \mu_{0}(0) \\ \Phi(\infty) & \text { value of } \Phi(t) \text { when } t \rightarrow \infty \\ \phi_{k} & k \text { order polynomial } \\ \Pi & \text { moment of order } k \text { of } P\end{array}$


$\theta \quad$ source in weight equation

$v \quad$ source in abscissas equation

$\rho \quad$ source in weighted abscissa

\section{Superscripts}

$\begin{array}{ll}a & \text { aggregation } \\ b & \text { breakage } \\ e & \text { exact solution of standard } \\ & \text { moment }\end{array}$

\section{Subscripts}

$\begin{array}{ll}\alpha & \text { quadrature point } \\ \beta & \text { abscissa } \\ \phi & \text { generalized moment } \\ \text { Fox } & \text { using Fox's heuristic scaling } \\ & \text { rule } \\ \text { OPT } & \text { using the optimization } \\ & \text { procedure } \\ \text { max } & \text { maximum } \\ \text { min } & \text { minimum } \\ R M S, 6 & \text { mean square relative error of } \\ & \text { the first six monomial } \\ & \text { moments }\end{array}$

\section{REFERENCES}

Alopaeus, V., Laakkonen, M. and Aittamaa, J., Numerical solution of moment-transformed population balance equation with fixed quadrature points. Chemical Engineering Science, 61(15), 4919-4929 (2006).

Anderson, E., Bai, Z., Bischof, C., Blackford, S., Demmel, J., Dongarra, J., Du Croz, J., Greenbaum, A., Hammarling, S., McKenney, A. and Sorensen, D., LAPACK Users' Guide. Society for Industrial and Applied Mathematics, Philadelphia, PA, Third Edition (1999).

Attarakih, M. M., Drumm, C. and Bart, H.-J., Solution of the population balance equation using the sectional quadrature method of moments (SQMOM). Chemical Engineering Science, 64 (4), 742-752 (2009).

Fox, R. O., Bivariate direct quadrature method of moments for coagulation and sintering of particle populations. Journal of Aerosol Science, 37(11), 1562-1580 (2006).

Friedlander, S. K., Smoke, Dust and Haze: Fundamentals of Aerosol Dynamics (2nd Ed.), Oxford University Press (2000).

Gautschi, W., Construction of Gauss-Christoffel quadrature formulas. Mathematics of Computa- tion, 22, 251-270 (1968).

Gautschi, W., Algorithm 726: ORTHPOL: A package of routines for generating orthogonal polynomials and Gauss-type quadrature rules. ACM Transactions on Mathematical Software, 20, 21-62 (1994).

Gautschi, W., Orthogonal Polynomials - Computation and Approximation. Oxford Science (2004).

Gordon, R., Error bounds in equilibrium statistical mechanics. Journal of Mathematical Physics, 9, 655-663 (1968).

Grosch, R., Marquardt, W., Briesen, H. and Wulkow, M., Generalization and numerical investigation of QMOM. AICHE J., 53, 207-227 (2006).

Hulburt, H. and Katz, S., Some problems in particle technology: A statistical mechanical formulation. Chemical Engineering Science, 19(8), 555-574 (1964).

Johnson, S. G., The NLopt nonlinear-optimization package. http://ab-initio.mit.edu/nlopt (2008).

Lage, P. L. C., Vaporização de Gotas Multicomponentes em Campos Convectivos e Radiantes. PhD Thesis, Programa de Engenharia Qumica, COPPE/UFRJ (1992). (In Portuguese).

Lage, P. L. C., On the representation of QMOM as a weighted-residual method - The dual-quadrature method of generalized moments. Computers \& Chemical Engineering, 35(11), 2186-2203 (2011).

Marchisio, D. L. and Fox, R. O., Solution of population balance equations using the direct quadrature method of moments. Journal of Aerosol Science, 36(1), 43-73 (2005).

Marechal, P. and Ye, J. J., Optimizing condition number. SIAM Journal on Optimization, 20(2), 935-947 (2009).

McCoy, B. J. and Madras, G., Analytical solution for a population balance equation with aggregation and fragmentation. Chemical Engineering Science, 58(13), 3049-3051 (2003).

McGraw, R., Description of aerosol dynamics by the quadrature method of moments. Aerosol Science and Technology, 27, 255-265 (1997).

Patil, D. P. and Andrews, J. R. G., An analytical solution to continuous population balance model describing floc coalescence and breakage - a special case. Chemical Engineering Science, 53(3), 599-601 (1998).

Ramkrishna, D., Population Balance - Theory and Applications to Particulate Systems in Engineering. Academic Press, San Diego, (2000).

Secchi, A. R., DASSLC: User's manual, a differentialalgebraic system solver. Technical report, UFRGS, Porto Alegre, RS/Brazil, (2007). Avaliable at http://www.enq.ufrgs.br/enqlib/numeric/DASSLC. 
Strumendo, M. and Arastoopour, H., Solution of PBE by MOM in finite size domains. Chemical Engineering Science, 63(10), 2624-2640 (2008).

Su, J., Gu, Z., Li, Y., Feng, S. and Xu, X. Y., Solution of population balance equation using quadrature method of moments with an adjustable factor. Chemical Engineering Science, 62(21), 5897-5911 (2007).

\section{APPENDICES}

\section{A) Population Balance Problem in a Semi-Infinite Domain}

The problem proposed by Patil and Andrews (1998), for which McCoy and Madras (2003) developed an analytical solution, was chosen to analyse the methods. The analytical solution is known for the initial condition:

$$
f(z, 0)=e^{-z}
$$

and for the aggregation frequency, breakage frequency and daughter distribution function defined as:

$$
b(z)=L z, \quad a\left(z^{\prime}, z\right)=K \quad P\left(z \mid z^{\prime}\right)=\frac{H\left(z^{\prime}-z\right)}{z^{\prime}}
$$

where $K$ was assumed to be 1 and $L$ is a constant calculated from a given $\Phi(\infty)$, defined below. The analytical solution is given by McCoy and Madras (2003):

$$
\begin{aligned}
f(t, z) & =\Phi^{2}(t) e^{-z \Phi(t)}, \quad \Phi(t) \\
& =\Phi(\infty)\left[\frac{1+\Phi(\infty) \tanh \left(\Phi(\infty) \frac{t}{2}\right)}{\Phi(\infty)+\tanh \left(\Phi(\infty) \frac{t}{2}\right)}\right]
\end{aligned}
$$

where $\Phi(\infty)$ is the value of $\Phi(t)$ in the steady-state which is given by:

$$
\Phi(\infty)=\sqrt{\frac{2 L}{K}} \frac{\sqrt{\mu_{1}(0)}}{\mu_{0}(0)}
$$

The regular moments are given by
Su, J., Gu, Z., Li, Y., Feng, S. and Xu, X. Y., An adaptive direct quadrature method of moment for population balance equations. AIChE J., 54, 2872-2887 (2008).

Wulkow, M., Gerstlauer, A. and Nieken, U., Modeling and simulation of crystallization processes using Parsival. Chemical Engineering Science, 56(7), 2575-2588 (2001).

$$
\mu_{k}(t)=\left[\frac{\Phi(\infty)+\tanh \left(\Phi(\infty) \frac{t}{2}\right)}{\Phi(\infty)[1+\Phi(\infty)]}\right]^{k-1} \Gamma(1+k)
$$

which, for $t=0$, simplifies to

$$
\mu_{k}(0)=\Gamma(1+k)
$$

\section{B) Population Balance Problem in a Finite Domain}

Lage (2011) defined a pure binary breakage problem using

$$
b(z)=z^{\gamma}, \quad P\left(z \mid z^{\prime}\right)=H\left(z^{\prime}-z\right) / z^{\prime}
$$

For a given value of $\gamma$, a source term can be defined in order for the following analytical solution be valid:

$f(z, t)=2-e^{-t}, z \in[0,1]$

whose moments are given by:

$\mu_{k}=\frac{2-e^{-t}}{k+1}$

For an integer value of $\gamma$, the breakage frequency $b(z)$ belongs to a finite dimensional polynomial space. Two cases were analysed with their corresponding PBE source terms:

- $\gamma=2 \Rightarrow R(z, t)=2 z^{2}\left(2-e^{-t}\right)-2\left(1-e^{-t}\right)$, and

- $\gamma=\frac{1}{3} \Rightarrow R(z, t)=7 e^{-t}-12+7\left(2-e^{-t}\right) z^{1 / 3}$. 